\title{
Three-dimensional hydrogel is suitable for targeted investigation of amoeboid migration of glioma cells
}

\author{
YUBAO HUANG ${ }^{1-3^{*}}$, LUQING TONG ${ }^{1,2^{*}}$, LI YI $^{1,2}$, CHEN ZHANG $^{1,2}$, LONG HAI $^{1,2}$, TAO LI $^{1,2}$, SHENGPING YU $^{1,2}$, \\ WEI WANG ${ }^{1,2}$, ZHENNAN TAO ${ }^{1,2}$, HAIWEN MA ${ }^{1,2}$, PEIDONG LIU ${ }^{1,2}$, YANG XIE ${ }^{1,2}$ and XUEJUN YANG ${ }^{1,2}$ \\ ${ }^{1}$ Department of Neurosurgery, Tianjin Medical University General Hospital; ${ }^{2}$ Laboratory of Neuro-Oncology, \\ Tianjin Neurological Institute; ${ }^{3}$ Department of Neurosurgery, Tianjin Haihe Hospital, Tianjin 300052, P.R. China
}

Received March 29, 2017; Accepted September 25, 2017

DOI: $10.3892 / \mathrm{mmr} .2017 .7888$

\begin{abstract}
Glioblastoma (GBM) invasion and migration are key biological behaviors leading to refractoriness to current therapies and infiltration into the non-tumor brain parenchyma. GBM cell migration is strongly dependent on tumor architecture in vivo, which is absent in traditional two-dimensional (2D) monolayer culture. The present study applied a three-dimensional (3D) hydrogel model to rebuild the tumor architecture in vitro. Treatment with NSC23766, a specific inhibitor of Ras-related C3 botulinum toxin substrate 1 (Rac1), inhibited the mesenchymal invasiveness however triggered the amoeboid motility called mesenchymal-amoeboid transition (MAT). Notably, NSC23766 stimulated U87 GBM cell migration in the 3D hydrogel. However, this compound inhibited cell motility in 2D monolayer culture without tumor architecture for MAT, suggesting the advantage of 3D hydrogel to investigate tumor cell invasion. Due to the inverse interaction of Rac1 and Ras homolog family member A (RhoA) signaling in the transition between mesenchymal and amoeboid morphology, simultaneous treatment of NSC23766 and Y27632 (selective Rho associated coiled-coil containing protein kinase 1 inhibitor), abolished U87 GBM cell migration through inhibiting MAT and amoeboid-mesenchymal transition. In addition, Y27632 induced integrin expression which gave rise to the focal adhesion to facilitate the mesenchymal invasion. The results of the present study demonstrated that the 3D hydrogel was a preferable model in vitro to study tumor cell invasion and migration. The combined inhibition of Racl and RhoA signaling would be a promising strategy to suppress GBM invasion.
\end{abstract}

Correspondence to: Dr Xuejun Yang, Department of Neurosurgery, Tianjin Medical University General Hospital, 154 An-shan, Tianjin 300052, P.R. China

E-mail: ydenny@yahoo.com

*Contributed equally

Key words: three-dimensional, hydrogel, targeted investigation, amoeboid migration, glioma

\section{Introduction}

Glioblastoma (GBM; WHO grade IV) is the most common and lethal primary malignant intracranial tumor (1). The invasion and migration of GBM cells remain a significant cause of palindromia and mortality and pose challenges for locally directed therapies such as surgery and radiation therapy (2). Inhibiting the invasion and migration of GBM cells has become an attractive treatment strategy. Recent studies have demonstrated that individual cells of certain cancers, such as GBM, have two different interconvertible patterns of movement: A mesenchymal pattern driven by Rac GTPase activation and an amoeboid pattern driven by Rho GTPase activation $(3,4)$. Because the ability of GBM cells to switch between different patterns of movement might limit the effectiveness of single therapeutic agents targeting the migration and invasion of cancer cells, simultaneously inhibiting Rac signaling and Rho signaling may be an effective strategy for GBM therapy (5).

Every cell in the body, including cancer cells, is surrounded by an intricate microenvironment. The in vivo microenvironment provides physical support and strongly influences the morphology, migration, proliferation and function of the cells therein (4), but most publications on cancer cells is based on the traditional two-dimensional (2D) culture. This discrepancy may be an important reason for the failure of the translation of numerous seemingly successful preclinical studies into clinical applications. In recent years, an increasing number of cell biology studies have been dedicated to three-dimensional (3D) cultures in which the cells are fully surrounded by matrix material and nutrition, in contrast to $2 \mathrm{D}$ culture, where the cells grow on top of a stiff surface. The biodegradability and porosity of 3D hydrogel may enable cancer cells to move in both mesenchymal and amoeboid patterns (6).

In this study, we established the 3D hydrogel model in vitro to investigate GBM cell motility inside the tumor architecture. 3D hydrogel showed more advantages on studying mesenchymal-amoeboid transition (MAT) and amoeboid-mesenchymal transition (AMT) than the 2D monolayer culture. Simultaneous treatment of NSC23766 [specific Ras-related C3 botulinum toxin substrate 1 (Racl) inhibitor] and Y27632 (selective ROCK1 inhibitor) abrogated U87 GBM cells migration through inhibiting both MAT and AMT. Additionally, Y27632 induced integrin expression which gave 
rise to the focal adhesion to facilitate the mesenchymal invasion.

\section{Materials and methods}

Ethical approval. All procedures performed in studies involving human participants were in accordance with the ethical standards of the Institutional and/or National Research Committee and with the 1964 Helsinki Declaration and its later amendments or comparable ethical standards. Informed consent was obtained from all individual participants included in the study.

Cells, antibodies and reagents. Glioma cell line U87 was purchased from the Chinese Academy of Sciences Cell Bank (Shanghai, China). Dulbecco's Modified Eagle's Medium (DMEM) and fetal bovine serum (FBS) were purchased from Gibco (Grand Island, NY, USA). Lipofectamine-3000 ${ }^{\mathrm{TM}}$ and Alexa Fluor 488-labeled Goat Anti-Mouse IgG were purchased from Invitrogen (Carlsbad, CA, USA). The antibiotic G418 was purchased from Merck KGaA (Darmstadt, Germany). Plasmid pCMV-LifeAct-TagGFP2 and $\mu$-Slide Chemotaxis ${ }^{3 \mathrm{D}}$ were purchased from ibidi GmbH (Martinsried, Germany). The specific inhibitors of NSC23766 and Y27632 were purchased from Selleck Chemicals (Houston TX, USA). Recombinant SDF-1was purchased from R\&D Systems (Minneapolis, MN, USA). 3D life dextran-CD hydrogel was purchased from CELLENDS (Germany). Mouse anti-integrin $\alpha \mathrm{V} \beta 3$ monoclonal antibody was purchased from Abcam (Cambridge, MA, USA).

Cell culture and plasmid transfection. U87 cells were cultured in DMEM medium containing $10 \% \mathrm{FBS}$ at $37^{\circ} \mathrm{C}$ under a humidified atmosphere with $5 \% \mathrm{CO}_{2}$. Lipofectin-3000 ${ }^{\mathrm{TM}}$ was used for the transfection of the Plasmid pCMV-LifeAct-TagGFP2 for $6 \mathrm{~h}$. The entire process was performed according to the manufacturer's instructions. The cells were cultured with $800 \mu \mathrm{g} / \mathrm{ml}$ G418, and the result of transfection was examined using an Olympus IX71 fluorescence microscope (Tianjin Neurological Institute, Tianjin, China).

Targeted treatment and group assignments. U87 cells were separated into 4 groups: The NSC23766 (Rac1 targeted inhibitor, $100 \mu \mathrm{M}$ )-treated group; the Y-27632 (ROCK targeted inhibitor, $10 \mu \mathrm{M}$ )-treated group; the combined treatment group with these two inhibitors; and the control group.

Wound healing cell migration assays. U87 cells were seeded in 6-well dishes $\left(2 \times 10^{5}\right.$ cells/well). On the next day, an artificial wound was made with a sterile pipette tip. Targeted inhibitors were then added as assigned to each group. The cells were then incubated at $37^{\circ} \mathrm{C}$ with $5 \% \mathrm{CO}_{2}$. Images of the wound area $24 \mathrm{~h}$ after injury were captured using an inverted microscope.

$3 D$ cultures in hydrogel and the chemotaxis experiment. To prepare the hydrogel, the polymers, $\mathrm{pH}$ buffer, water, peptides, crosslinker and cell suspension with final a concentration of $10^{6} / \mathrm{ml}$ were mixed as described in the manufacturer's instructions. The final concentration of themaleimide and thiol groups reached $2.5 \mathrm{mmol} / \mathrm{l}$, thus making the gel strength similar to brain tissue.
To observe the transformation of cell morphology in the 3D hydrogel, the cells were treated with targeted inhibitors according to their group assignments, and images were collected at $40-\mathrm{sec}$ intervals at $37^{\circ} \mathrm{C}$ with $5 \% \mathrm{CO}_{2}$ using laser confocal microscopy (PerkinElmer, Inc., Waltham, MA, USA).

Cells in the hydrogel system prepared as described above were pretreated with the targeted inhibitors for $3 \mathrm{~h}$. Chemotaxis experiments have been done once as previously described $(7,8)$. The hydrogel system was injected into the observation channel of $\mu$-Slide Chemotaxis ${ }^{3 \mathrm{D}}$, the complete medium containing SDF-1 $(100 \mathrm{nmol} / \mathrm{ml})$ was injected into the left reservoir, and the complete medium without SDF-1 was injected into the right reservoir. For time-lapse microscopic analyses, cells were cultured at $37^{\circ} \mathrm{C}$ with $5 \% \mathrm{CO}_{2}$ for $12 \mathrm{~h}$. Images were collected at 2-min intervals with a living cells workstation (Nikon Ti-E; Nikon Corp., Tokyo, Japan).

Immunofluorescence staining. The integrin expression in the cells that had been observed for morphology translation was evaluated by immunofluorescence staining. Cells were fixed with $4 \%$ paraformaldehyde for $15 \mathrm{~min}$, permeabilized with $0.5 \%$ Triton X-100 for $20 \mathrm{~min}$, and blocked with $0.5 \%$ BSA in PBS for $1 \mathrm{~h}$. Immunofluorescence staining was performed by incubating the hydrogel system with a mouse anti-integrin $\alpha \mathrm{V} \beta 3$ monoclonal antibody (1:50) overnight at $4^{\circ} \mathrm{C}$ and with Alexa Fluor 488-labeled goat anti-mouse secondary antibody $(1: 1,000)$ for $2 \mathrm{~h}$ on the next day. Immunofluorescence was visualized, and the images were captured with an Olympus IX71 fluorescence microscope (Tianjin Neurological Institute).

Patient data analysis. Patient data and gene expression datasets were obtained using the R2 microarray analysis and visualization platform (http://hgserver1.amc.nl/cgi-bin/r2/main.cgi). All prognosis analyses were conducted online, and all data and $\mathrm{P}$ values (log-rank test) were downloaded. Kaplan-Meier analysis and the resulting survival curves were performed using GraphPad Prism (version 6.0; GraphPad Software, Inc., La Jolla, CA, USA). All cutoff values for separating high and low expression groups were determined by the online R2 database algorithm. Medians were used for separating high and low expression groups online.

Statistical analysis. All quantified data represent an average of at least triplicate experiments unless otherwise indicated, and standard deviations were calculated. All statistical analyses were performed using GraphPad Prism 6.0. Comparisons among groups were determined by multiple comparisons of ordinary one-way ANOVA, where $\mathrm{P}<0.05$ was significant.

\section{Results}

Racl and Ras homolog family member A (RhoA) were closely related to the prognosis of patients. We evaluated the correlation between Rac1/RhoA and overall survival (OS) through R2 genomics analysis and the visualization platform database. Medians were used for separating high and low expression groups by the online R2 database algorithm. Rac1 was highly expressed in 137 of the 273 cases of glioma. Racl expression was negatively correlated with the patient OS in French's data ( $\mathrm{P}=1.6 \mathrm{e}-0.6$; Fig. 1A). Similarly, RhoA was upregulated 

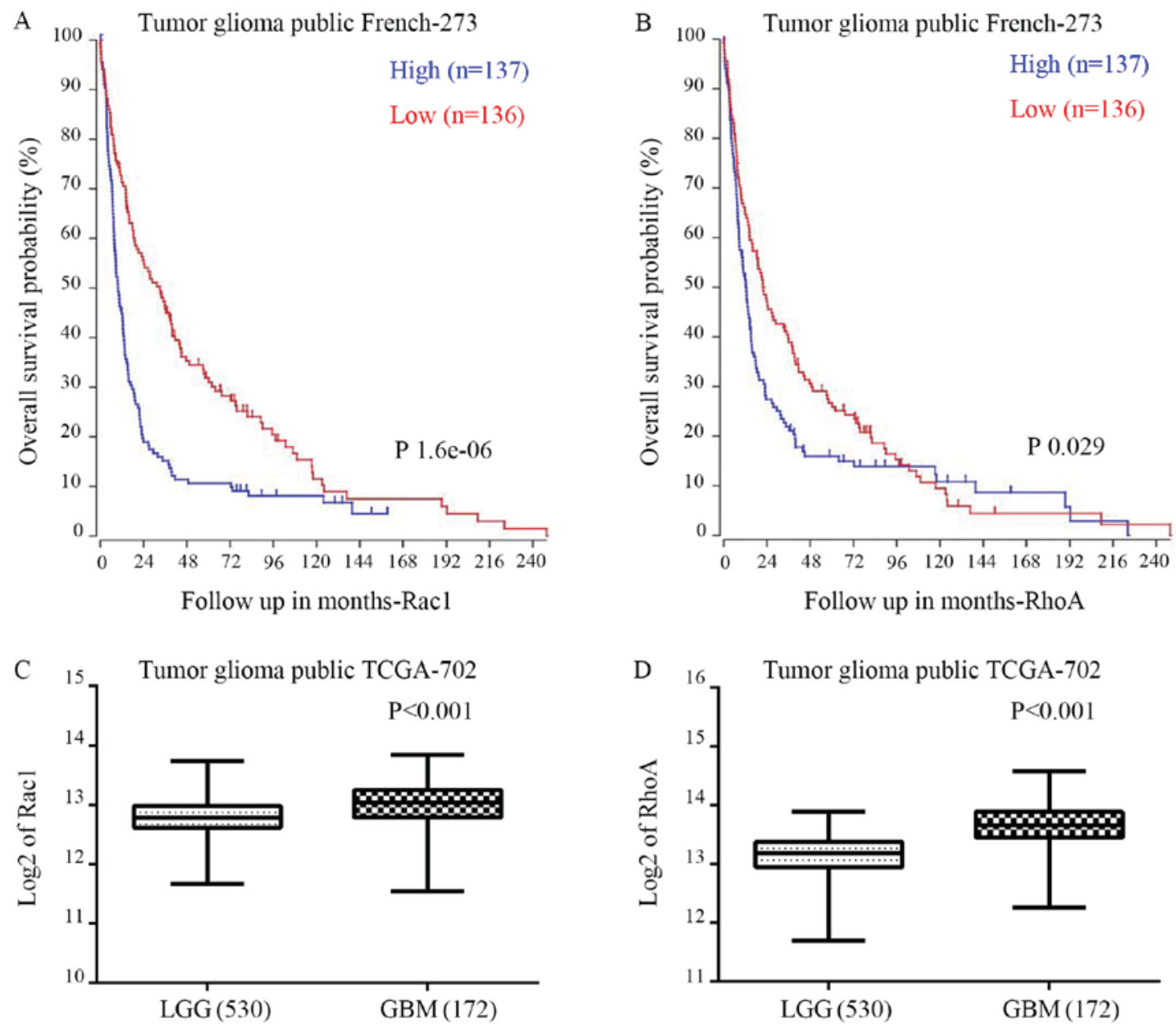

Figure 1. High Rac1 and RhoA expression are prognostic indicators of poor survival in glioblastoma patients. (A and B) Kaplan-Meier analysis of overall survival probability for the French database with the log-rank test. (C and D) Box plot of Rac1/RhoA expression levels in LGG and GBM. Rac1, Ras-related C3 botulinum toxin substrate 1; RhoA, Ras homolog family member A; LGG, lower grade glioma; GBM, glioblastoma.

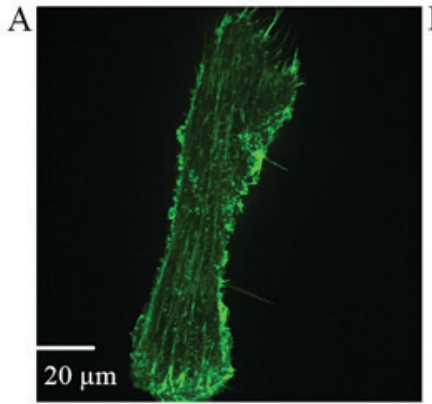

Flat in 2D monolayer

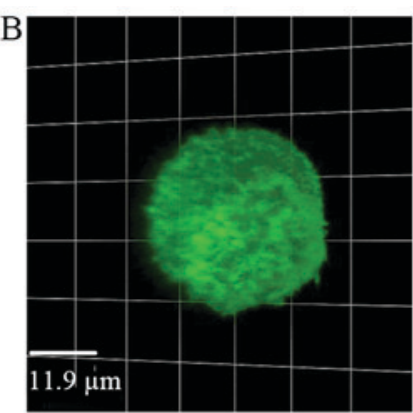

Round-like in 3D hydrogel

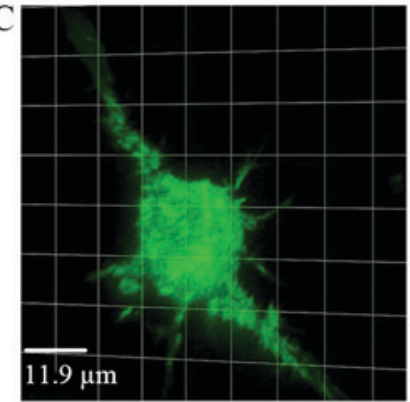

Spindle in 3D hydrogel

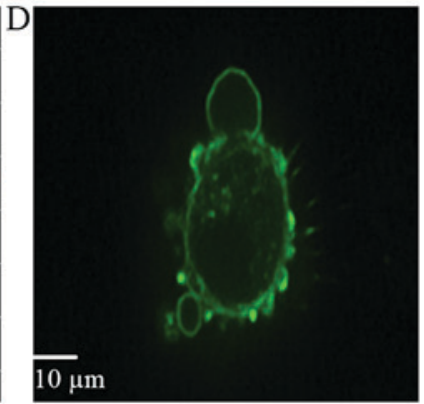

Blebbing in 3D monolayer

Figure 2. The morphology of U87 cells in 2D monolayer and 3D hydrogel (x1,000). Cells transfected with plasmid pCMV-LifeAct-TagGFP2 were cultured both in 2D monolayer and 3D hydrogel. (A) U87 glioma cells presented as flat and protruded several broad lamellipodium without obvious polarity in 2D monolayer culture. In 3D hydrogel culture, cells presented as (B) round-like or (C) spindle. (D) In particular sections, blebs next to round-like cells can be seen. 2D, two-dimensional; 3D, three-dimensional.

in 137 cases among the 273 patients. RhoA also showed negative correlation with the patient OS (P=0.029; Fig. 1B). Accordingly, in contrast to lower grade gliomas (LGG), both Racl and RhoA were significantly upregulated in GBM patients according to TCGA data (Fig. 1C and D).

The morphology of cells in $2 D$ monolayer and $3 D$ hydrogel. To investigate cell morphology in detail, U87 GBM cells were transfected with plasmid pCMV-LifeAct-TagGFP2 and the morphology was observed with a confocal microscopy. In the 2D monolayer culture, U87 cells presented as flat and protruded several broad lamellipodium without polarity (Fig. 2A). In the 3D hydrogel culture, the cells exhibited as spindle or round-like (Fig. 2B-D). Most spindle cells contained one or two spindly protrusion and harbored obvious polarity. Blebs around the round-like cells were observed (Fig. 2D). 

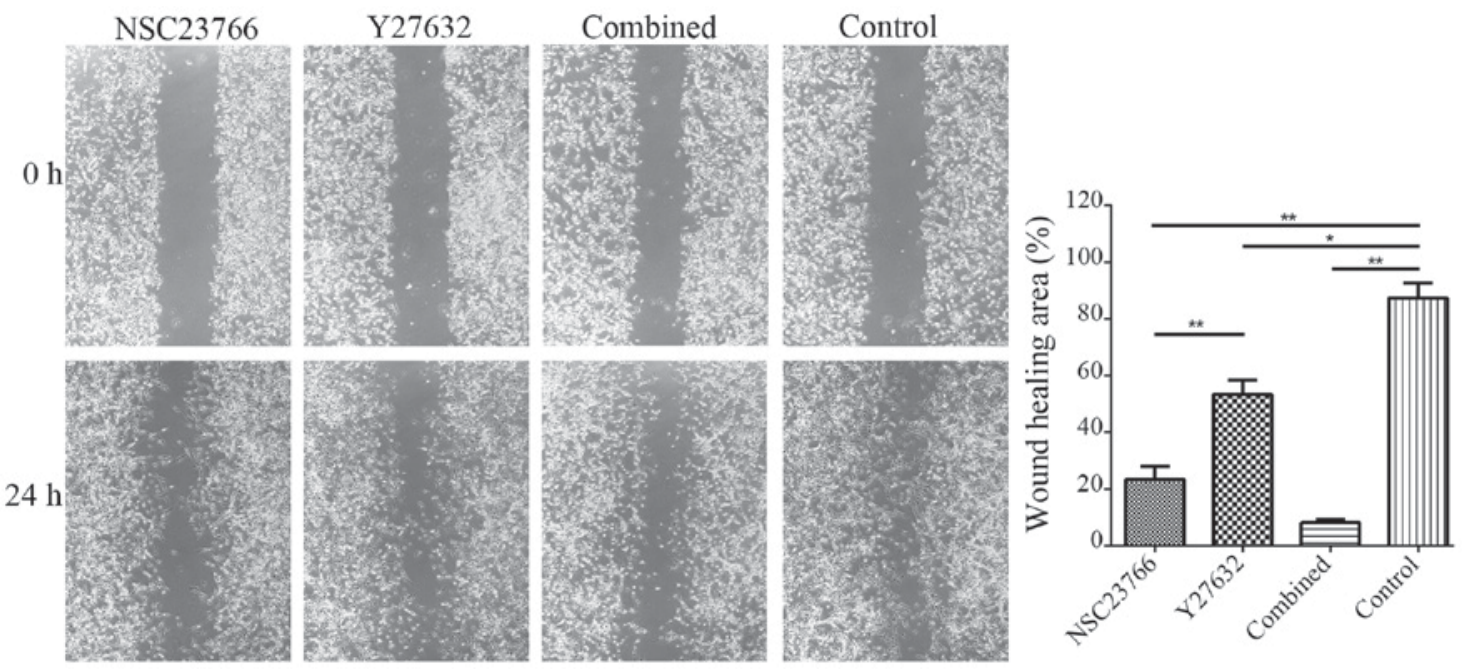

Figure 3. Targeted inhibited migration of U87 cells in 2D monolayer $(\mathrm{x} 1,000)$. A classic wound healing assay for assessing the migration of the targeted inhibited U87 cells by single or jointly agents. There were significant differences between each experimental group and the control group $\left({ }^{*} \mathrm{P}<0.05\right.$ and $\left.{ }^{* *} \mathrm{P}<0.01\right)$, and the cells of the NSC23766-treated group were much slower than were cells of the Y27632-treated group ("* $\mathrm{P}<0.01)$. 2D, two-dimensional.

Migration-targeted inhibitions in the $2 D$ monolayer. Considering the mesenchymal pattern driven by Rac GTPase activation and the amoeboid pattern driven by Rho GTPase activation, NSC23766 (specific Rac1 inhibitor) and Y27632 (selective ROCK1 inhibitor) were introduced to treat U87 glioma cells in the traditional 2D monolayer culture. The migration of U87 cells to the wound area was analyzed at $24 \mathrm{~h}$ after injury and treatment. As a result, the wound healed $23.35 \pm 8.10 \%$ in the NSC23766-treated group, 53.38 $\pm 8.80 \%$ in the Y27632-treated group, $8.18 \pm 1.93 \%$ in the combined treatment group, and $87.38 \pm 9.30 \%$ in the control group. Cells in the combined treatment group migrated significantly more slowly than did the control group $(\mathrm{P}<0.01$; Fig. 3$)$. This finding revealed that inhibiting both Racl and RhoA abolished the wound healing of U87 GBM cells.

Targeted inhibited invasion and migration in the $3 D$ hydrogel. Because the tumor architecture strongly influences the morphology, invasion, and migration of cells (9), the efficiency of targeted inhibition was investigated in 3D hydrogel. U87 cells were treated with targeted inhibitors according to their assigned groups, and the morphology of these cells was observed with a confocal microscope at 40 -sec intervals (Fig. 4). The morphology of the cells underwent MAT transformed gradually from spindle- to round-like, and the protrusion disappeared (Fig. 4B). In contrast, the morphology of the cells underwent AMT transformed gradually from round-like to spindle-shaped, and the protrusion extended gradually (Fig. 4A).

Single cell migration towards SDF-1 was observed by living cells workstation. U87 cells were pretreated with targeted inhibitors for $3 \mathrm{~h}$. Under the chemotaxis of SDF-1 for $12 \mathrm{~h}$, the invasion and migration of U87 cells were assessed by live cell tracking in $\mu$-slide chemotaxis chambers as described in the materials and methods section. Images were collected at 2-min intervals with time-lapse microscopy, and all cell trajectories were recorded using the ImageJ software (Fig. 4A). In addition, movement velocity and distance were calculated. The movement velocity and 12-h movement distance of the different treatment groups were $1.253 \pm 0.282 \mu \mathrm{m} / \mathrm{min}$, $0.663 \pm 0.178,0.567 \pm 0.110$ and $0.917 \pm 0.166 \mu \mathrm{m} / \mathrm{min}$ (Fig. $4 \mathrm{C}$ ) and $824.645 \pm 184.608,402.703 \pm 179.196,149.149 \pm 63.765$ and $603.085 \pm 109.441 \mu \mathrm{m}$ (Fig. 4D), respectively. The differences were significant between the experimental groups and the control group $(\mathrm{P}<0.05$ for each). Interestingly, NSC23766 treated cells moved much faster than were cells in other groups in the 3D hydrogel $(\mathrm{P}<0.05$; Fig. $4 \mathrm{C}$ and $\mathrm{D})$. However, NSC23766 treated cells moved slower than other group cells in the $2 \mathrm{D}$ monolayer $(\mathrm{P}<0.05$; Fig. 3$)$.

Integrin expression in targeted inhibited cells. The formation of focal adhesion mediated by integrin is an important process during the invasion of glioma cells. Integrin $\alpha \mathrm{V} \beta 3$ expression was detected by immunofluorescence staining to assess the adhesion of U87 cells. The integrin $\alpha \mathrm{V} \beta 3$ expression of the Y27632-treated group was much higher than in the other groups. The integrin $\alpha \mathrm{V} \beta 3$ expression of the Y27632-treated group and the combined treatment group was much lower than in the control group (Fig. 5).

\section{Discussion}

GBM is the most common intracranial malignant tumor, and it has a poor prognosis anda powerful capacity for invasion and migration $(2,10)$. GBM cells have two different interconvertible patterns of movement: The mesenchymal pattern driven by Rac GTPase activation and the amoeboid pattern driven by Rho GTPase activation $(3,4,11)$. Mesenchymal pattern is the main motility model in the brain white matter with a slower speed $(0.1-1 \mu \mathrm{m} / \mathrm{min})(12)$. The tumor cells with mesenchymal motility pattern presented the spindle morphology and the invasion dependent on the enzymatic hydrolysis of the extracellular matrix (ECM) (13). Rac1 promotes the polymerization from G-actins to F-actins and serves as the key molecule of mesenchymal movement $(14,15)$. Amoeboid pattern is the main motility model in subpial, subependymal and extravascular space with a faster speed (a maximum of $4.0 \mu \mathrm{m} / \mathrm{min}$ can be achieved) (12). The cells with amoeboid 
A

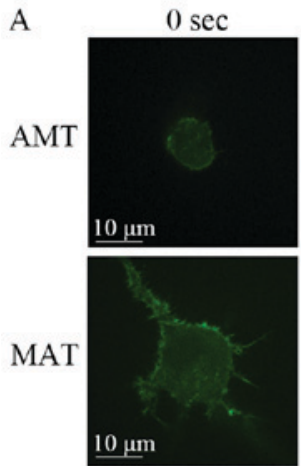

$40 \mathrm{sec}$

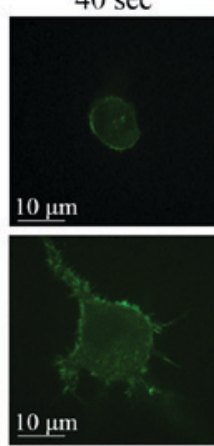

$80 \mathrm{sec}$

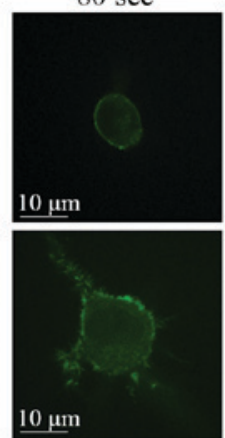

$120 \mathrm{sec}$
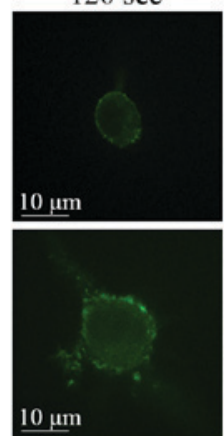

$160 \mathrm{sec}$
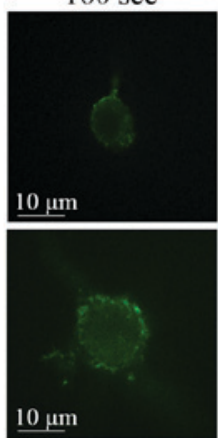

$200 \mathrm{sec}$
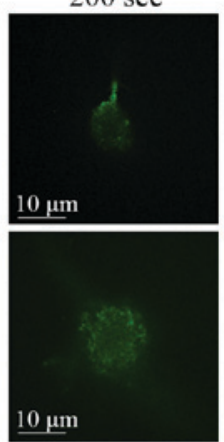

B
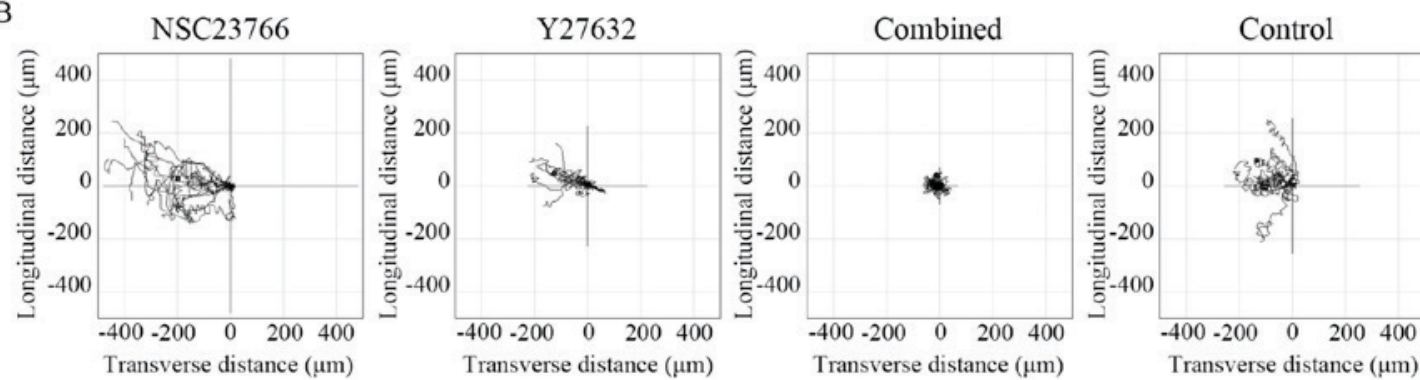

C

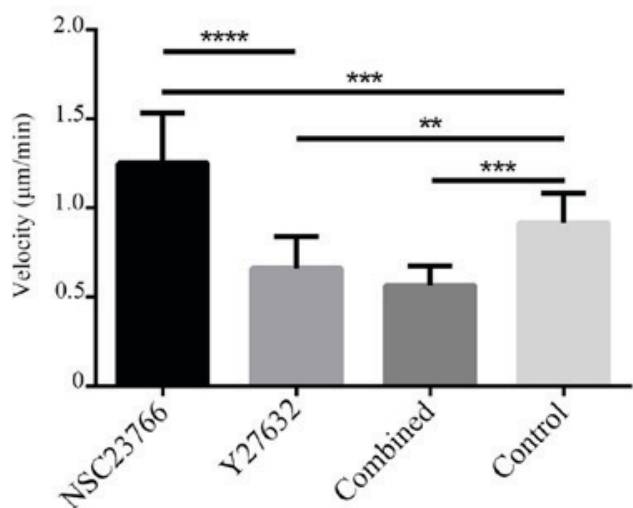

D

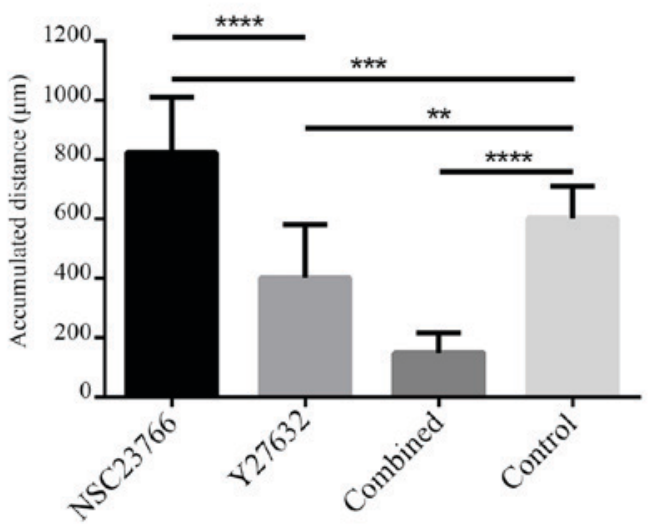

Figure 4. Targeted inhibited invasion and migration of U87 cells in 3D hydrogel. (A) MAT was observed when U87 cells were treated with NSC23766 and AMT was observed when treated with Y27632 (x1,000). (B) U87 cells treated with targeted inhibitors separately or jointly were assessed by live cell tracking in $\mu$-slide chemotaxis chambers as described in the Materials and methods section. All cell trajectories were recorded by ImageJ software. (C) The movement velocity and (D) 12-h movement distance of different treatment groups were calculated, and there were significant differences between the experimental

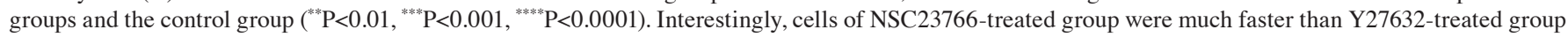
$\left(\mathrm{C}\right.$ and $\left.\mathrm{D},{ }^{* * * *} \mathrm{P}<0.0001\right)$. 3D, three-dimensional; MAT, mesenchymal-amoeboid transition; AMT, amoeboid-mesenchymal transition.

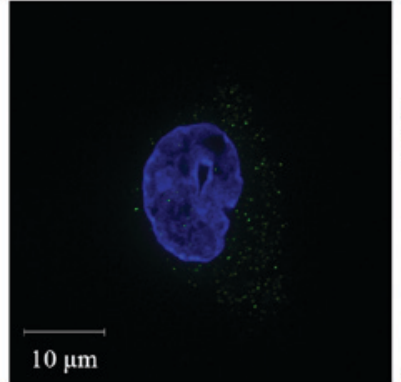

NSC23766

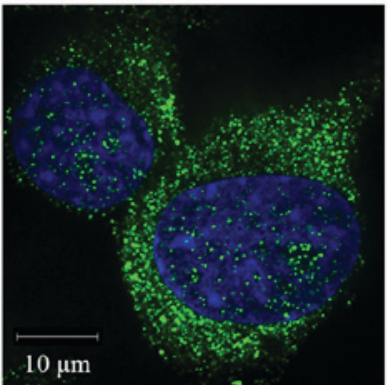

Y27632

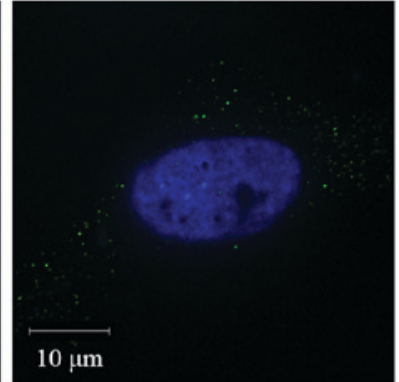

Combined

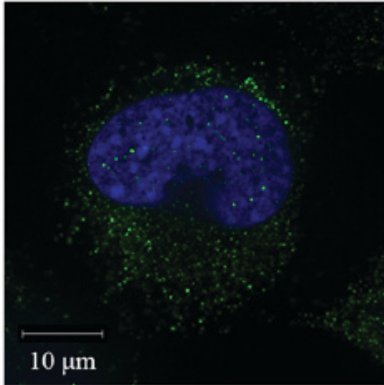

Control

Figure 5. Integrin $\alpha \mathrm{V} \beta 3$ expression of targeted inhibited cells (x1,000). Integrin $\alpha \mathrm{V} \beta 3$ expression of U87 cells was detected by immunofluorescence staining. The integrin $\alpha \mathrm{V} \beta 3$ expression of the Y27632-treated group was much higher than that of the other groups, whereas the expression of the NSC23766-treated group and the combined treatment group were much lower than the control group.

motility pattern exhibited a round-like morphology and the migration dependent on the actomyosin contraction driven by RhoA rather than the polarization driven by Rac1 $(12,16)$. The induction of actomyosin contractility via RhoA-ROCK 
signaling leads to blebbing, thus causing amoeboid migration, but it also regulates tail retraction in mesenchymal migration $(2,16,17)$. In this study, single-locus analysis revealed that patients with a relatively higher Rac1/RhoA level harbored shorter OS (Fig. 1A). Additionally, theRac1/RhoA level was increased in GBM compared with the lower-grade glioma (Fig. 1B).

Consistent with Stephen Paget's 128-year-old 'seed-and-soil' hypothesis, the microenvironment in vivo provides a physical support and strongly influences the morphology, migration, proliferation and function of cells therein (9), whereas most studies of cancer cells are currently based on the traditional 2D culture. Cell properties including extending vesicle, invasion, mesenchymal and amoeboid movement are unable to be thoroughly studied in the 2D monolayer. Despite the advantages of animal models on studying tumorigenesis and progression, it is difficult to detect aimed molecules and trace the cell motion in vivo (18). Even the organotypic brain slice cannot be used for the morphological and tracing research of a single cell (19). Cells in 3D cultures mimicking the microenvironment in vivo are fully surrounded by matrix material, in contrast to $2 \mathrm{D}$ culture, where cells are grown on top of a stiff surface. The biodegradability and porosity of the 3D hydrogel enable cancer cells to move in both mesenchymal and amoeboid patterns $(20,21)$. In this study, 3D life dextran-CD hydrogelwas utilized for U87 GBM cell culture. Because cell morphology and motor pattern can be affected by the stiffness of the environment (22), the final concentrations of maleimide and thiol groups were made up to $2.5 \mathrm{mmol} / \mathrm{l}$; thus, the gel strength is similar to brain tissue. The results suggested that U87 cells had two diverse patterns, i.e., spindle- and round-like (Fig. 2B-D), in 3D hydrogel, whereas they presented a flat pattern in the $2 \mathrm{D}$ monolayer (Fig. 2A).

Because the conversion between different patterns of movement might limit the efficiency of single therapeutic agents, combined therapy targeting Racl and RhoA would be a promising strategy to restrain the invasion and migration of GBM cells $(23,24)$. Rac1-WAVE signaling has been shown to promote cytoskeletal reorganization and invadopodium formation (17). ROCK is a downstream molecular target of RhoA, which induces actomyosin contraction and causes amoeboid migration. Thus, Rho activation promotes cell contraction and hinders mesenchymal movement by modulating Rac-GAP ARHGAP22 signaling $(12,16)$. NSC23766 is a specific inhibitor of the binding and activation of Rac1 GTPase and does not inhibit the closely related targets, Cdc42 or RhoA. In this study, NSC23766 was used to inhibit Rac1 signaling. Because RhoA contributes to both amoeboid and mesenchymal modes of migration (25), Y27632 (selective ROCK1 inhibitor) was selected to inhibit RhoA-ROCK signaling.

In the 2D monolayer, the cells in the NSC23766-treated group migrated more slowly than did the cells in both the control group and the Y27632-treated group in the wound healing assay $(\mathrm{P}<0.05$ for each, Fig. 3). Interestingly, the cells of the NSC23766 treated group invaded and migrated faster than the cells of the Y27632 treated group and the control group in $3 \mathrm{D}$ hydrogel $(\mathrm{P}<0.05, \mathrm{P}<0.05$; Fig. 4D). This result is consistent with the phenomenon that some tumor cells migrate in an amoeboid pattern with a higher speed in vivo (12). Otherwise, it probably revealed the reason that numerous seemingly successful preclinical studies based on the $2 \mathrm{D}$ model failed in translating into clinical applications. At the same time, MAT was observed in the NSC23766 treated group and AMT was observed in theY27632 treated group in 3D hydrogel (Fig. 4A), both of which cannot be seen in the 2D monolayer. Additionally, the results of combined inhibition targeting Racl and RhoA indicated that both the movement velocity and 12-h movement distance were significantly decreased (Fig. 4C and D).

In fact, the average movement velocity of U87 cells treated by NSC23766 was not the same as the tumor cells moving in an amoeboid pattern with the maximum of $4.0 \mu \mathrm{m} / \mathrm{min}$. Several facts might contribute to this difference. Firstly, $1.253 \pm 0.282 \mu \mathrm{m} / \mathrm{min}$ was the general speed of 15 cells, and not all of the cells were moving in an amoeboid pattern. Secondly, the stiffness of the ECM influences the invasion and migration (26). The soft hydrogel lacks stiffness and allows the glioma cells to move quickly in an amoeboid pattern. Last but not least, the porosity of the hydrogel is an important environmental factor for cell migration. Cell migration is fastest at pore diameters that match or are slightly smaller than the cells; migration speed decreases in large pore size matrices due to the loss of cell-matrix interactions, but pore sizes much smaller than the cell diameter trap cells in a physical cage and reduce cell migration (27). In this hydrogel, the average diameter was slightly smaller than the cell size with a final concentration of $2.5 \mathrm{mmol} / \mathrm{l}$ of maleimide and thiol groups, and it is difficult to avoid the fluctuation of diameter within a certain range.

Integrin $\alpha \mathrm{V} \beta 3$ was identified as a driver of an aggressive and metastatic tumor phenotype $(28,29)$. Here, the integrin $\alpha \mathrm{V} \beta 3$ expression of targeted inhibited U87 cells was detected by immunofluorescence staining to assess the adhesion. The results showed that the integrin $\alpha \mathrm{V} \beta 3$ expression of the Y27632-treated group was much higher than those of the other groups. The integrin $\alpha \mathrm{V} \beta 3$ expressions of the NSC23766-treated group and combined treatment group were much lower than that of control group (Fig. 5). This result verified the fact that Rac signaling activates inversely with Rho signaling.

In conclusion, we mainly investigated the invasion and migration of U87 glioma cells and targeted therapy in 3D hydrogel. The results showed that the U87 cells treated with NSC23766 moved much faster than did the control cells in the $3 \mathrm{D}$ chemotactic assay, whereas they moved much more slowly than did the control cells in the 2D wound healing assay. This result probably revealed the reason why numerous seemingly successful preclinical studies based on 2D model have failed to be translated into clinical applications. The effective inhibition of invasion and migration by the combined targeting Rac1 and RhoA suggested that combined targeted therapy may be a promising strategy for preventing invasion and migration of GBM cells.

\section{Acknowledgements}

This study was supported by the National Natural Science Foundation of China (nos. 81272782 and 81472352); and the Tianjin Research Program of Application Foundation and Advanced Technology (no. 15JCZDJC36200). 


\section{References}

1. Stupp R, Hegi ME, Mason WP, van den Bent MJ, Taphoorn MJ, Janzer RC, Ludwin SK, Allgeier A, Fisher B, Belanger K, et al: Effects of radiotherapy with concomitant and adjuvant temozolomide versus radiotherapy alone on survival in glioblastoma in a randomised phase III study: 5-year analysis of the EORTC-NCIC trial. Lancet Oncol 10: 459-466, 2009.

2. Friedl P and Wolf K: Tumour-cell invasion and migration: Diversity and escape mechanisms. Nat Rev Cancer 3: 362-374, 2003.

3. Sanz-Moreno V, Gadea G, Ahn J, Paterson H, Marra P, Pinner S, Sahai E and Marshall CJ: Rac activation and inactivation control plasticity of tumor cell movement. Cell 135: 510-523, 2008.

4. Rao SS, Bentil S, DeJesus J, Larison J, Hissong A, Dupaix R, Sarkar A and Winter JO: Inherent interfacial mechanical gradients in 3D hydrogels influence tumor cell behaviors. PloS One 7: e35852, 2012.

5. Parri M and Chiarugi P: Rac and Rho GTPases in cancer cell motility control. Cell Commun Signal 8: 23, 2010.

6. Jiguet Jiglaire C, Baeza-Kallee N,Denicolaï E, Barets D, Metellus P, Padovani L, Chinot O, Figarella-Branger D and Fernandez C: Ex vivo cultures of glioblastoma in three-dimensional hydrogel maintain the original tumor growth behavior and are suitable for preclinical drug and radiation sensitivity screening. Exp Cell Res 321: 99-108, 2014.

7. Zengel P, Nguyen-Hoang A, Schildhammer C, Zantl R, Kahl V and Horn E: $\mu$-Slide chemotaxis: A new chamber for long-term chemotaxis studies. BMC Cell Biol 12: 21, 2011.

8. Gouwy M, De Buck M, Pörtner N, Opdenakker G, Proost P, Struyf S and Van Damme J: Serum amyloid A chemoattracts immature dendritic cells and indirectly provokes monocyte chemotaxis by induction of cooperating CC and CXC chemokines. Eur J Immunol 45: 101-112, 2015.

9. Thiele J, Ma Y, Bruekers SM, Ma S and Huck WT: 25th anniversary article: Designer hydrogels for cell cultures: A materials selection guide. Adv Mater 26: 125-147, 2014.

10. Lu KV, Chang JP, Parachoniak CA, Pandika MM, Aghi MK, Meyronet D, Isachenko N, Fouse SD, Phillips JJ, Cheresh DA, et al: VEGF inhibits tumor cell invasion and mesenchymal transition through a MET/VEGFR2 complex. Cancer Cell 22: 21-35, 2012.

11. Mikheeva SA, Mikheev AM, Petit A, Beyer R, Oxford RG, Khorasani L, Maxwell JP, Glackin CA, Wakimoto H, GonzálezHerrero I, et al: TWIST1 promotes invasion through mesenchymal change in human glioblastoma. Mol Cancer 9: 194, 2010.

12. Symons M and Segall JE: Rac and Rho driving tumor invasion: Who's at the wheel? Genome Biol 10: 213, 2009.

13. Yamazaki D, Kurisu S and Takenawa T: Involvement of Rac and Rho signaling in cancer cell motility in $3 \mathrm{D}$ substrates. Oncogene 28 1570-1583, 2009.

14. Zhong J, Paul A, Kellie SJ and O'Neill GM: Mesenchymal migration as a therapeutic target in glioblastoma. J Oncol 2010: 430142, 2010.

15. Wuichet K and Søgaard-Andersen L: Evolution and diversity of the Ras superfamily of small GTPases in prokaryotes. Genome Biol Evol 7: 57-70, 2014.
16. Haga RB and Ridley AJ: Rho GTPases: Regulation and roles in cancer cell biology. Small GTPases 7: 207-221, 2016.

17. Murali A and Rajalingam K: Small Rho GTPases in the control of cell shape and mobility. Cell Mol Life Sci 71: 1703-1721, 2014.

18. Rape AD and Kumar S: A composite hydrogel platform for the dissection of tumor cell migration at tissue interfaces. Biomaterials 35: 8846-8853, 2014.

19. Ren B, Yu S, Chen C, Wang L, Liu Z, Wu Q, Wang L, Zhao K and Yang X: Invasion and anti-invasion research of glioma cells in an improved model of organotypic brain slice culture. Tumori 101: 390-397, 2015.

20. Flemming A: Cancer: Multifunctional nanodevice reverses drug resistance. Nat Rev Drug Discov 14: 309, 2015

21. Shea LD, Woodruff TK and Shikanov A: Bioengineering the ovarian follicle microenvironment. Annu Rev Biomed Eng 16: 29-52, 2014.

22. Souza GR, Molina JR, Raphael RM, Ozawa MG, Stark DJ, Levin CS, Bronk LF, Ananta JS, Mandelin J, Georgescu MM, et al: Three-dimensional tissue culture based on magnetic cell levitation. Nat Nanotechnol 5: 291-296, 2010.

23. Reymond N, Im JH, Garg R, Vega FM, Borda d'Agua B, Riou P, Cox S, Valderrama F, Muschel RJ and Ridley AJ: Cdc42 promotes transendothelial migration of cancer cells through $\beta 1$ integrin. J Cell Biol 199: 653-668, 2012.

24. Navarro-Lérida I, Pellinen T, Sanchez SA, Guadamillas MC, Wang Y, Mirtti T, Calvo E and Del Pozo MA: Rac1 nucleocytoplasmic shuttling drives nuclear shape changes and tumor invasion. Dev Cell 32: 318-334, 2015.

25. Sadok A, McCarthy A, Caldwell J, Collins I, Garrett MD, Yeo M, Hooper S, Sahai E, Kuemper S, Mardakheh FK and Marshall CJ: Rho kinase inhibitors block melanoma cell migration and inhibit metastasis. Cancer Res 75: 2272-2284, 2015.

26. Mishima T, Naotsuka M, Horita Y, Sato M, Ohashi K and Mizuno K: LIM-kinase is critical for the mesenchymal-to-amoeboid cell morphological transition in 3D matrices: Biochem Biophys Res Commun 392: 577-581, 2010.

27. Panková K, Rösel D, Novotný M and Brábek J: The molecular mechanisms of transition between mesenchymal and amoeboid invasiveness in tumor cells. Cell Mol Life Sci 67: 63-71, 2010

28. Desgrosellier JS, Barnes LA, Shields DJ, Huang M, Lau SK, Prévost N, Tarin D, Shattil SJ and Cheresh DA: An integrin alpha(v)beta(3)-c-Src oncogenic unit promotes anchorage-independence and tumor progression. Nat Med 15: 1163-1169, 2009.

29. Seguin L, Kato S, Franovic A, Camargo MF, Lesperance J, Elliott KC, Yebra M, Mielgo A, Lowy AM, Husain H, et al: An integrin $\beta_{3}$-KRAS-RalB complex drives tumour stemness and resistance to EGFR inhibition. Nat Cell Biol 16: 457-468, 2014.

This work is licensed under a Creative Commons

Attribution-NonCommercial-NoDerivatives 4.0 International (CC BY-NC-ND 4.0) License. 